\title{
Bopp-Podolsky black holes and the no-hair theorem
}

\author{
R. R. Cuzinatto ${ }^{1,2}$, C. A. M. de Melo ${ }^{2,3}$, L. G. Medeiros $^{3,4, a}$, B. M. Pimentel ${ }^{3}$, P. J. Pompeia ${ }^{5}$ \\ ${ }^{1}$ Department of Physics, McGill University, Ernest Rutherford Physics Building, 3600 University Street, Montreal, QC H3A 2T8, Canada \\ ${ }^{2}$ Instituto de Ciência e Tecnologia, Universidade Federal de Alfenas, Rod. José Aurélio Vilela (BR 267), Km 533, n. 11999, Poços de Caldas, MG \\ CEP 37701-970, Brazil \\ ${ }^{3}$ Instituto de Física Teórica, Universidade Estadual Paulista, Rua Bento Teobaldo Ferraz 271 Bloco II, P.O. Box 70532-2, São Paulo, SP CEP \\ 01156-970, Brazil \\ ${ }^{4}$ Escola de Ciência e Tecnologia, Universidade Federal do Rio Grande do Norte, Campus Universitário, s/n-Lagoa Nova, Natal, RN CEP 59078-970, \\ Brazil \\ ${ }^{5}$ Departamento de Física, Instituto Tecnológico de Aeronáutica, Praça Mal. Eduardo Gomes 50, São José dos Campos, SP CEP 12228-900, Brazil
}

Received: 6 November 2017 / Accepted: 4 January 2018 / Published online: 19 January 2018

(C) The Author(s) 2018. This article is an open access publication

\begin{abstract}
Bopp-Podolsky electrodynamics is generalized to curved space-times. The equations of motion are written for the case of static spherically symmetric black holes and their exterior solutions are analyzed using Bekenstein's method. It is shown that the solutions split up into two parts, namely a non-homogeneous (asymptotically massless) regime and a homogeneous (asymptotically massive) sector which is null outside the event horizon. In addition, in the simplest approach to Bopp-Podolsky black holes, the non-homogeneous solutions are found to be Maxwell's solutions leading to a Reissner-Nordström black hole. It is also demonstrated that the only exterior solution consistent with the weak and null energy conditions is the Maxwell one. Thus, in the light of the energy conditions, it is concluded that only Maxwell modes propagate outside the horizon and, therefore, the no-hair theorem is satisfied in the case of BoppPodolsky fields in spherically symmetric space-times.
\end{abstract}

\section{Introduction}

It was just 1 year after the proposal of General Relativity by Einstein in 1915 [1] that the first analytical solution to his gravitational field equations was obtained. Schwarzschild [2] proposed a spherical symmetric solution for the gravitational/metric field produced by a point mass. In the same year, Reissner [3] proposed a solution to a charged point mass, which was 2 years later reconsidered by Nordström [4] in a spherical coordinate system known today as the Reissner-Nordström solution. These solutions were the first ones predicting the existence of event horizons for very compact objects. This work paved the way for a whole new area

a e-mail: leogmedeiros@gmail.com of research in gravitation commonly known as black hole (BH) physics.

The decades of 1960 and 1970 witnessed a boom of interest in this area. In 1963, Kerr [5] presented his solution for a spinning mass, a result that was generalized by Newman 2 years later with the introduction of electric charge to the rotating body $[6,7]$. A few years later, important developments related to the interaction between matter and gravitational fields were achieved. In this line of research, a relevant contribution was given by Israel [8] in 1967, when he proposed the first version of the no-hair theorem/conjecture for the spherically symmetric black holes. This result was soon extended to include rotating and charged $\mathrm{BHs}[9,10]$ and a final version of this theorem states that an exterior solution of a $\mathrm{BH}$ is completely characterized by its mass, electric charge and angular momentum. All other features of particles ("hair") have no contribution to the gravitational properties of the black hole once these particles are inside the event horizon. This theorem has been demonstrated for many cases [11-13] and for different theories of gravity [14-16] but several results suggest its validity is limited - see $[17,18]$ and the references therein. As a matter of fact, haired solutions appear for complex Proca fields in the vicinity of spinning BHs [19]; in addition, Ref. [20] presents the possibility that super-radiance could amplify quantum effects making these hairs potentially detectable by gravitational-wave observatories.

The real Proca field is a particularly interesting case, studied by Bekenstein in [11]. He analyzed BHs in the presence of a massive vector field (henceforth called Proca black holes). This is a compelling case which verifies the no-hair theorem. Bekenstein built an ingenious argument to show that the massive field cannot propagate outside the event horizon 
without making use of an analytical explicit solution. ${ }^{1}$ This way, no information as regards the mass of the Proca field can be obtained by an observer outside the BH. This case is in contrast with the massless (Maxwell) vectorial field, which is clearly known to propagate outside the event horizon. So, at this point, one could ask: How can Maxwell fields propagate outside the event horizon whilst Proca field cannot?

The two most significant differences between Proca and Maxwell fields lie in the fact that the latter is a massless gauge invariant field, while the former is a massive non-gauge invariant field. Thus, the question above may be reformulated as: Can gauge invariance and the mass/massless property be the keystone for the difference concerning field propagation between these two cases? In order to answer this question, we will consider an extension of Maxwell's theory proposed in the early 1940s by Bopp and Podolsky [26,27], known today as Bopp-Podolsky electrodynamics, or shortly Podolsky electrodynamics. The Lagrangian of this theory is characterized, in addition to the usual Maxwell term, by a term depending on the second derivative of the gauge field, leading to fourth-order field equations. Although higher order theories usually suffer from instabilities (ghost at the quantum level), they can be avoided in Podolsky's case by using the concept of a Lagrange anchor [28]. Moreover, Podolsky electrodynamics presents unique properties that make it worth analyzing. For instance, this theory has been proven to be the only second order gauge theory for the $U(1)$ group to preserve the linearity of the field equations [29]. Also, the solution of the field equations shows the Podolsky field splits in two modes: a massive mode and a massless one. These two properties render the Podolsky field one of the most promising candidates to properly address the question raised before. It is important to emphasize that these properties of Podolsky electrodynamics are valid in flat space-time. Thus, it is essential to verify that they are still valid in curved space-time. Although some aspects of this theory in curved space-time are found in the literature [30-33], these two properties will be analyzed in some detail here.

Our intention in this paper is to understand the propagation of vector fields outside the event horizon for Podolsky black holes. In particular, we are interested in verifying if the no-hair theorem remains valid. For this purpose, we will analyze the properties of the electrostatic spherically symmetric solution of Podolsky electrodynamics in curved space-time. In Sect. 2, we will analyze how Podolsky electrodynamics is properly generalized to curved space-time, considering its gauge invariance and linearity properties. In Sects. 3 and 4, we shall investigate the properties of the exterior solution using Bekenstein's approach. In Sect. 5, we reanalyze these

\footnotetext{
${ }^{1}$ Until now, there are only perturbative [21,22] and numerical [23-25] solutions to the Proca black hole.
}

properties scrutinizing the null and weak energy conditions. Final remarks are presented in Sect. 6.

\section{Podolsky electrodynamics in curved space-time}

From a formal point of view, the Podolsky electrodynamics Lagrangian $\mathcal{L}_{m}$ is built under the following assumptions:

1. $\mathcal{L}_{m}$ must be invariant under Lorentz transformations (in flat space-times);

2. $\mathcal{L}_{m}$ must be gauge invariant under $U(1)$ symmetry group, i.e. under a transformation of the type $A_{\mu} \rightarrow A_{\mu}+\partial_{\mu} \alpha$;

3. $\mathcal{L}_{m}$ must be quadratic in the gauge field and its derivatives resulting in linear field equations;

4. $\mathcal{L}_{m}$ is dependent on the gauge field and its first two derivatives.

The authors of Ref. [29] followed the approach developed by Utiyama [34] to show that $\mathcal{L}_{m}$ is a combination of the Maxwell Lagrangian and terms of the form $\mathcal{L}_{m} \sim \partial$. $F^{*}$..$F^{*}$ " in Minkowski space-time, where the repeated symbol "." indicates index contraction. If we analyze all possible contractions of the indices, we verify that only three non-null and non-trivially equivalent terms remain:

$\mathcal{L}_{m}^{(1)}=\partial_{\beta} F^{\alpha \beta} \partial_{\gamma} F_{\alpha}{ }^{\gamma}$,

$\mathcal{L}_{m}^{(2)}=\partial_{\beta} F^{\alpha \gamma} \partial_{\gamma} F_{\alpha}{ }^{\beta}$

$\mathcal{L}_{m}^{(3)}=\partial_{\beta} F^{\alpha \gamma} \partial^{\beta} F_{\alpha \gamma}$.

It is not difficult to verify that $\mathcal{L}_{m}^{(2)}$ is equivalent to $\mathcal{L}_{m}^{(1)}$ (up to a surface term) and that $\mathcal{L}_{m}^{(3)}$ can be obtained from $\mathcal{L}_{m}^{(2)}$ when the Bianchi identity [29] is taken into account. Therefore, in flat space-time Podolsky electrodynamics is completely described by the Lagrangian

$\mathcal{L}_{m}^{\text {flat }}=-\frac{1}{4} F^{\alpha \beta} F_{\alpha \beta}+\frac{a^{2}}{2} \partial_{\beta} F^{\alpha \beta} \partial_{\gamma} F_{\alpha}{ }^{\gamma}$.

Note that the positive sign in the second term allows the factor $\frac{1}{a}$ to be interpreted as a mass parameter $[35,36]$. We consider the metric to have signature $(+,-,-,-)$. Lagrangian $\mathcal{L}_{m}^{\text {flat }}$ was proposed originally in the early 1940s by Bopp [26] and Podolsky [27].

The next step is to generalize the approach presented in [29] to curved space-times. To this end, in assumption 1 above, Lorentz invariance is replaced by general covariance and a minimal coupling prescription is considered, i.e. the following mappings apply: $\eta_{\mu \nu} \rightarrow g_{\mu \nu}, \partial_{\mu} \rightarrow \nabla_{\mu}$. As a consequence, assumption 4 demands the Lagrangian to be of the form $\mathcal{L}_{m}(A, \nabla A, \nabla \nabla A)$. Next we impose the group symmetry condition (assumption 2 ) under $U(1)$, which leads 
to

$$
\begin{gathered}
\frac{\partial \mathcal{L}_{m}}{\partial A_{\mu}} \delta A_{\mu}+\frac{\partial \mathcal{L}_{m}}{\partial\left(\nabla_{\nu} A_{\mu}\right)} \nabla_{\nu}\left(\delta A_{\mu}\right) \\
+\frac{\partial \mathcal{L}_{m}}{\partial\left(\nabla_{\lambda} \nabla_{\nu} A_{\mu}\right)} \nabla_{\lambda} \nabla_{\nu}\left(\delta A_{\mu}\right)=0,
\end{gathered}
$$

where $\delta A_{\mu}=\nabla_{\mu} \alpha$. By considering the functional independence of $\alpha$ and its covariant derivatives ${ }^{2}$ we find

$\frac{\partial \mathcal{L}_{m}}{\partial A_{\mu}} \nabla_{\mu} \alpha=0$

$\frac{\partial \mathcal{L}_{m}}{\partial\left(\nabla_{\nu} A_{\mu}\right)} \nabla_{\nu} \nabla_{\mu} \alpha=0$

$\frac{\partial \mathcal{L}_{m}}{\partial\left(\nabla_{\lambda} \nabla_{\nu} A_{\mu}\right)} \nabla_{\lambda} \nabla_{\nu} \nabla_{\mu} \alpha=0$.

Analogously to what occurs in the case of flat space-time, Eq. (2) states that $\mathcal{L}_{m}$ does not depend explicitly on $A_{\mu}$. Then from Eq. (3) we see $\mathcal{L}_{m}$ depends on $\nabla_{\mu} A_{v}$ only through an antisymmetric combination, given by

$F_{\mu \nu} \equiv \nabla_{\mu} A_{\nu}-\nabla_{\nu} A_{\mu}$

and derivatives of $F_{\mu \nu}$. Note that the antisymmetry of $\nabla A$ was established in view of the identity $\nabla_{\nu} \nabla_{\mu} \alpha=\nabla_{\mu} \nabla_{\nu} \alpha$.

Now we implement assumptions 1 and 3 and verify that, besides the Maxwell term, only combinations of the form $\mathcal{L} \sim \nabla . F^{*} \nabla . F^{*}$ are allowed. As before, only three non-null and non-trivially equivalent terms remain,

$\mathcal{L}_{m}^{(1)}=\nabla_{\beta} F^{\alpha \beta} \nabla_{\gamma} F_{\alpha}{ }^{\gamma}$,

$\mathcal{L}_{m}^{(2)}=\nabla_{\beta} F^{\alpha \gamma} \nabla_{\gamma} F_{\alpha}^{\beta}$,

$\mathcal{L}_{m}^{(3)}=\nabla_{\beta} F^{\alpha \gamma} \nabla^{\beta} F_{\alpha \gamma}$.

Direct substitution of these terms into Eq. (4) shows that this equation is satisfied for all terms.

Finally, we have to check if $\mathcal{L}_{m}^{(1)}, \mathcal{L}_{m}^{(2)}$ and $\mathcal{L}_{m}^{(3)}$ are equivalent to each other. By using the covariant version of the Bianchi identity,

$\nabla_{\beta} F_{\alpha \gamma}+\nabla_{\alpha} F_{\gamma \beta}+\nabla_{\gamma} F_{\beta \alpha}=0$

we show that $\mathcal{L}_{m}^{(3)}=2 \mathcal{L}_{m}^{(2)}$. On the other hand, $\mathcal{L}_{m}^{(1)}$ and $\mathcal{L}_{m}^{(2)}$ are related through ${ }^{3}$

$$
\begin{aligned}
\mathcal{L}_{m}^{(2)}= & \nabla_{\gamma}\left(F_{\alpha}{ }^{\beta} \nabla_{\beta} F^{\alpha \gamma}\right)-\nabla_{\beta}\left(F_{\alpha}{ }^{\beta} \nabla_{\gamma} F^{\alpha \gamma}\right) \\
& +R_{\sigma \beta} F^{\sigma \alpha} F_{\alpha}{ }^{\beta}+R_{\alpha \sigma \beta \gamma} F^{\sigma \gamma} F^{\alpha \beta}+\mathcal{L}_{m}^{(1)} .
\end{aligned}
$$

The first two terms on the right-hand side are terms of the form $\nabla_{\mu} V^{\mu}$, hence they constitute surface terms. However,

2 The requirement of functional independence of $\alpha$ and its ordinary derivatives leads to a system equivalent to Eqs. (2)-(4).

${ }^{3}$ In this manuscript, the Riemann tensor is defined by $R_{\mu \nu \kappa}^{\sigma} \equiv$ $\partial_{\nu} \Gamma_{\mu \kappa}^{\sigma}-\partial_{\kappa} \Gamma_{\mu \nu}^{\sigma}+\Gamma_{\alpha \nu}^{\sigma} \Gamma_{\mu \kappa}^{\alpha}-\Gamma_{\alpha \kappa}^{\sigma} \Gamma_{\mu \nu}^{\alpha}$. the non-commutativity of covariant derivatives imply the presence of two extra terms consisting of non-minimal couplings between the field strength and the Riemann tensor. These extra terms show that $\mathcal{L}_{m}^{(1)}$ and $\mathcal{L}_{m}^{(2)}$ are not equivalent.

Thus, we conclude that the Podolsky electrodynamics in curved space-times is given by the combination

$\mathcal{L}_{m}=-\frac{1}{4} F^{\alpha \beta} F_{\alpha \beta}+\frac{a^{2}}{2} \nabla_{\beta} F^{\alpha \beta} \nabla_{\gamma} F_{\alpha}^{\gamma}+\frac{b^{2}}{2} \nabla_{\beta} F^{\alpha \gamma} \nabla^{\beta} F_{\alpha \gamma}$.

Equation (7) is useful in rewriting Eq. (8) as

$$
\begin{aligned}
\mathcal{L}_{m}= & -\frac{1}{4} F^{\alpha \beta} F_{\alpha \beta}+\frac{\left(a^{2}+2 b^{2}\right)}{2} \nabla_{\beta} F^{\alpha \beta} \nabla_{\gamma} F_{\alpha}{ }^{\gamma} \\
& +b^{2}\left(R_{\sigma \beta} F^{\sigma \alpha} F_{\alpha}{ }^{\beta}+R_{\alpha \sigma \beta \gamma} F^{\sigma \gamma} F^{\alpha \beta}\right) .
\end{aligned}
$$

This expression shows Podolsky electrodynamics obtained by Utiyama's approach presents two terms with non-minimal coupling in addition to the usual term obtained by the minimal coupling prescription in Eq. (1). It is interesting to note that non-minimally coupled terms of this form also emerge in other contexts such as vacuum polarization in a curved background [37] and quantization of the Einstein-Maxwell theory [38].

Hence, we can deal with Einstein-Podolsky system from two different perspectives: we can choose a minimal coupling in Eq. (1) which leads to Eq. (9) with $b=0$, or we can choose a more general approach where we also consider the non-minimally coupled terms preserving conditions 1 to 4 above and characterized by $b \neq 0$. The next sections are dedicated to the analyses of these two cases in the context of black holes with spherical symmetry.

\subsection{Field equations}

We consider Einstein-Podolsky action ${ }^{4}$

$S=\frac{1}{16 \pi} \int \mathrm{d}^{4} x \sqrt{-g}\left[-R+4 \mathcal{L}_{m}\right]$,

where $\mathcal{L}_{m}$ is given by Eq. (8). The corresponding field equations are obtained from the variation of $S$ with respect to the fields $A_{\mu}$ and $g^{\mu \nu}$. From the variation with respect to $A_{\mu}$ we obtain Podolsky equations in curved space-time:

$\nabla_{v}\left[F^{\mu \nu}-\left(a^{2}+2 b^{2}\right) H^{\mu \nu}+2 b^{2} S^{\mu \nu}\right]=0$,

where $H^{\mu \nu}$ and $S^{\mu \nu}$ are antisymmetric tensors defined as

$$
\begin{aligned}
H^{\mu \nu} & \equiv \nabla^{\mu} K^{v}-\nabla^{v} K^{\mu}, \\
S^{\mu \nu} & \equiv F^{\mu \sigma} R_{\sigma}{ }^{v}-F^{v \sigma} R_{\sigma}^{\mu}+2 R_{\sigma \beta}^{\mu \nu} F^{\beta \sigma},
\end{aligned}
$$

\footnotetext{
${ }^{4}$ We take $G=1=c$ throughout the paper.
} 
with

$K^{\mu} \equiv \nabla_{\gamma} F^{\mu \gamma}$

Variation of $S$ with respect to $g^{\mu \nu}$ leads to the Einstein equations,

$R_{\mu \nu}-\frac{1}{2} g_{\mu \nu} R=8 \pi T_{\mu \nu}=8 \pi\left(T_{\mu \nu}^{M}+T_{\mu \nu}^{a}+T_{\mu \nu}^{b}\right)$,

where

$$
\begin{aligned}
T_{\mu \nu}^{M}= & \frac{1}{4 \pi}\left[F_{\mu \sigma} F_{\nu}^{\sigma}+g_{\mu \nu} \frac{1}{4} F^{\alpha \beta} F_{\alpha \beta}\right], \\
T_{\mu \nu}^{a}= & \frac{a^{2}}{4 \pi}\left[g_{\mu \nu} F_{\beta}^{\gamma} \nabla_{\gamma} K^{\beta}+\frac{g_{\mu \nu}}{2} K^{\beta} K_{\beta}\right. \\
& \left.+2 F_{(\mu}^{\alpha} \nabla_{\nu)} K_{\alpha}-2 F_{(\mu}^{\alpha} \nabla_{\alpha} K_{\nu)}-K_{\mu} K_{\nu}\right], \\
T_{\mu \nu}^{b}= & \frac{b^{2}}{2 \pi}\left[-\frac{1}{4} g_{\mu \nu} \nabla^{\beta} F^{\alpha \gamma} \nabla_{\beta} F_{\alpha \gamma}+F_{(\mu}^{\gamma} \nabla^{\beta} \nabla_{\beta} F_{\nu) \gamma}\right. \\
& \left.+F_{\gamma(\mu} \nabla_{\beta} \nabla_{v)} F^{\beta \gamma}-\nabla_{\beta}\left(F_{\gamma}^{\beta} \nabla_{(\mu} F_{\nu)}^{\gamma}\right)\right] .
\end{aligned}
$$

The notation (...) indicates symmetrization with respect to indices $\mu \nu$. Moreover, the trace of the energy-momentum tensor is

$$
\begin{aligned}
T= & \frac{\left(a^{2}+2 b^{2}\right)}{4 \pi} K^{\beta} K_{\beta}+\frac{b^{2}}{4 \pi} \\
& \times\left[2 \nabla_{\beta}\left(F^{\gamma \alpha} \nabla^{\beta} F_{\alpha \gamma}\right)+F^{\gamma \mu} S_{\mu \gamma}\right] .
\end{aligned}
$$

Now we consider this system of field equations in the particular case of (static) spherical symmetry. The line element can be written as

$\mathrm{d} s^{2}=e^{\nu(r)} \mathrm{d} t^{2}-e^{\lambda(r)} \mathrm{d} r^{2}-r^{2} \mathrm{~d} \theta^{2}-r^{2} \sin ^{2} \theta \mathrm{d} \phi^{2}$,

while the field strength is given by

$F_{\mu v}=E(r)\left[\delta_{\mu}^{1} \delta_{v}^{0}-\delta_{\mu}^{0} \delta_{v}^{1}\right]$

So, the system is completely characterized by three functions: the radial electric field $E(r)$ and the gravitational components $v(r)$ and $\lambda(r)$.

In view of this parametrization we rewrite Eq. (11) as

$E-\left(a^{2}+2 b^{2}\right) \partial_{1} K_{0}+2 b^{2} S_{10}=C \frac{e^{\frac{(v+\lambda)}{2}}}{r^{2}}$,

where $C$ is an arbitrary integration constant and

$$
\begin{aligned}
K_{0} & =\frac{e^{\frac{\nu-\lambda}{2}}}{r^{2}} \partial_{1}\left(r^{2} e^{-\frac{(\nu+\lambda)}{2}} E\right), \\
S_{10} & =E e^{-\lambda}\left(\frac{\nu^{\prime}-\lambda^{\prime}}{r}\right) .
\end{aligned}
$$

The prime denotes the derivative with respect to the radial coordinate, e.g. $v^{\prime} \equiv \partial_{r} v \equiv \partial_{1} v$. In flat space-time the spherically symmetric solution to the electromagnetic sector, Eq. (22), depends on $A_{\mu}=\left(A_{0}, 0,0,0\right)$, which is a function of $x^{1}=r$ solely. It is the one due to Podolsky [27]:

$A_{0}=\frac{C_{1}}{r}-\frac{C_{2} e^{-\frac{r}{r_{p}}}}{r}$

with $r_{p}^{2}=a^{2}+2 b^{2}$.

The non-null components of Eq. (15) are given by

$$
\begin{aligned}
& e^{-\lambda}\left(\frac{\lambda^{\prime}}{r}-\frac{1}{r^{2}}\right)+\frac{1}{r^{2}}=8 \pi T_{0}^{0}, \\
& -e^{-\lambda}\left(\frac{v^{\prime}}{r}+\frac{1}{r^{2}}\right)+\frac{1}{r^{2}}=8 \pi T_{1}^{1}, \\
& -\frac{1}{4 r} e^{-\lambda}\left[\left(v^{\prime}-\lambda^{\prime}\right)\left(2+r v^{\prime}\right)+2 r v^{\prime \prime}\right]=8 \pi T_{2}^{2},
\end{aligned}
$$

where

$$
\begin{aligned}
T_{0}^{0}= & -\frac{g^{00} g^{11}}{8 \pi}\left\{E\left[E-2\left(a^{2}+2 b^{2}\right) \partial_{1} K_{0}+4 b^{2} S_{10}\right]\right. \\
& \left.+\frac{a^{2} K_{0}^{2}}{g^{11}}+2 b^{2} g^{11}\left[\left(\frac{K_{0}}{g^{11}}+\frac{2 E}{r}\right)^{2}+\frac{2 E^{2}}{r^{2}}\right]\right\}, \\
T_{1}^{1}= & -\frac{g^{00} g^{11}}{8 \pi}\left\{E\left[E-2\left(a^{2}+2 b^{2}\right) \partial_{1} K_{0}+4 b^{2} S_{10}\right]\right. \\
& \left.-\frac{a^{2} K_{0}^{2}}{g^{11}}-2 b^{2} g^{11}\left[\left(\frac{K_{0}}{g^{11}}+\frac{2 E}{r}\right)^{2}+\frac{2 E^{2}}{r^{2}}\right]\right\}, \\
T_{2}^{2}= & \frac{g^{00} g^{11}}{8 \pi}\left\{E^{2}-a^{2}\left[2 E \partial_{1} K_{0}-\frac{K_{0}^{2}}{g^{11}}\right]+\frac{2 b^{2} K_{0}^{2}}{g^{11}}\right. \\
& \left.-4 b^{2} g^{11}\left[\left(\frac{K_{0}}{g^{11}}+\frac{2 E}{r}\right)^{2}+\frac{E S_{10}}{2 g^{11}}+\frac{2 E^{2}}{r^{2}}\right]\right\} .
\end{aligned}
$$

The requirement of spherical symmetry turns Eq. (19) for the trace of the energy-momentum tensor into

$$
\begin{aligned}
T= & \frac{\left(a^{2}-2 b^{2}\right)}{4 \pi} g^{00} K_{0}^{2}+\frac{b^{2}}{\pi} g^{00} g^{11} E\left(\partial_{1} K_{0}-\frac{4 K_{0}}{r}\right) \\
& +\frac{b^{2}}{\pi} g^{00}\left(g^{11} E\right)^{2}\left[\frac{3}{2} \frac{v^{\prime}-\lambda^{\prime}}{r}-\frac{6}{r^{2}}\right] .
\end{aligned}
$$

This concludes our calculations of the field equations for the Podolsky BH. The solution to these equations depends on physically meaningful boundary conditions. The first is to recover the Minkowski space-time far from the source, i.e. when $r \rightarrow \infty$, which shall be defined as $r_{\infty}$. The second boundary is the surface of the horizon, located by coordinate $r=r_{H}$, appearing in the black hole solution we will study next. 


\section{Analyzis of Podolsky black hole exterior solution in the case $b=0$}

In this section we study in detail the exterior solution of a spherical BH in Podolsky electrodynamics in the particular case corresponding to $b=0$. We show that the only non-null exterior solution is identical to Maxwell's, which means that the $\mathrm{BH}$ is of the Reissner-Nordström type.

\subsection{Bekenstein's technique}

By taking $b=0$ in Eq. (11), contracting this equation with $A_{\mu}$ and integrating the result in the 4-volume exterior to $\mathrm{BH}$, we get

$\int A_{\mu} \partial_{\nu}\left[\sqrt{-g}\left(F^{\mu \nu}-a^{2} H^{\mu \nu}\right)\right] \mathrm{d}^{4} x=0$.

Notice that under spherical symmetry the 4-volume is limited by the horizon $r_{H}$, by $r_{\infty}(r \rightarrow \infty)$ and by the past and future infinite times $t \rightarrow \pm \infty$. Integration by parts leads to

$I_{M}-I_{a}-\oint \sqrt{-g} A_{\mu}\left(F^{\mu \nu}-a^{2} H^{\mu \nu}\right) \mathrm{d} S_{\nu}=0$,

where

$$
\begin{aligned}
I_{M} & =\int \sqrt{-g} F^{\mu \nu} \partial_{\nu} A_{\mu} \mathrm{d}^{4} x, \\
I_{a} & =a^{2} \int \sqrt{-g} H^{\mu \nu} \partial_{\nu} A_{\mu} \mathrm{d}^{4} x,
\end{aligned}
$$

with

$$
\begin{aligned}
I_{a}= & a^{2} \int \sqrt{-g} g^{\mu \beta} g^{\nu \alpha}\left(\partial_{\nu} A_{\mu}\right)\left(\partial_{\beta} K_{\alpha}\right) \mathrm{d}^{4} x \\
& +a^{2} \int K_{\beta} \partial_{\alpha}\left(\sqrt{-g} g^{\mu \beta} g^{\nu \alpha} \partial_{\nu} A_{\mu}\right) \mathrm{d}^{4} x \\
& -a^{2} \oint \sqrt{-g} g^{\mu \beta} g^{\nu \alpha} K_{\beta} \partial_{\nu} A_{\mu} \mathrm{d} S_{\alpha} .
\end{aligned}
$$

Therefore Eq. (33) is written as the sum of three integrals:

$$
I_{1}+I_{2}+I_{3}=0
$$

where

$$
\begin{aligned}
I_{1}= & I_{M}-a^{2} \int K_{\beta} \partial_{\alpha}\left(\sqrt{-g} g^{\mu \beta} g^{\nu \alpha} \partial_{\nu} A_{\mu}\right) \mathrm{d}^{4} x \\
& -a^{2} \int \sqrt{-g} g^{\mu \beta} g^{\nu \alpha}\left(\partial_{\nu} A_{\mu}\right)\left(\partial_{\beta} K_{\alpha}\right) \mathrm{d}^{4} x, \\
I_{2}= & a^{2} \oint \sqrt{-g} g^{\mu \beta} g^{\nu \alpha} K_{\beta} \partial_{\nu} A_{\mu} \mathrm{d} S_{\alpha}, \\
I_{3}= & -\oint\left[\sqrt{-g} A_{\mu}\left(F^{\mu \nu}-a^{2} H^{\mu \nu}\right)\right] \mathrm{d} S_{\nu},
\end{aligned}
$$

The next step is to impose spherical symmetry: $A_{\mu}=$ $\left(A_{0}(r), 0,0,0\right)$. Consequently, the three integrals become

$$
\begin{aligned}
& I_{1}=\int \sqrt{-g} g^{00}\left[-g^{11} E^{2}+\left(a K_{0}\right)^{2}\right] \mathrm{d}^{4} x, \\
& I_{2}=a^{2}\left[\int_{r_{H}}+\int_{r_{\infty}}\right] \sqrt{-g} g^{00} g^{11} K_{0} E \mathrm{~d} S_{1}, \\
& I_{3}=\left[\int_{r_{H}}+\int_{r_{\infty}}\right] \frac{\sqrt{-g} A_{0}}{g_{00} g_{11}}\left[E-a^{2} \partial_{1} K_{0}\right] \mathrm{d} S_{1},
\end{aligned}
$$

where the notation with $r_{H}$ and $r_{\infty}$ means that the integrals are performed on surfaces of fixed $r$. Notice that the flux integrals in Eqs. (36) and (37) are different from zero only on surfaces where $r$ is constant.

Let us analyze the properties of $I_{2}$ and $I_{3}$ at $r_{\infty}$. When $r \rightarrow \infty$ the space-time becomes flat (Minkowski) so that

$\sqrt{-g} \mathrm{~d} S_{1} \approx r^{2} \mathrm{~d} S$

and $A_{0}$ is given by Eq. (25). Using this in Eqs. (39) and (40) shows that the integrals over the surfaces with $r=r_{\infty}$ appearing in $I_{2}$ and $I_{3}$ are null.

The case for the integrals over the surfaces with $r=r_{H}$ is more complicated. First, we recall that the trace of the energy-momentum tensor given by Eq. (32) with $b=0$ is

$T=\frac{a^{2}}{4 \pi} g^{00}\left(K_{0}\right)^{2}$.

This is a scalar with physical meaning - it is associated with the energy of the system -, hence it must be finite on the horizon. On the other hand, $g^{00}\left(r_{H}\right) \rightarrow \infty$. Consequently, $K_{0}$ must approach zero at least at the same rate as $\sqrt{g_{00}}$ in order to guarantee a finite value for $T$ on the horizon. In this case,

$$
I_{2} \sim a^{2} \int_{r_{H}} E r^{2} \sin \theta \sqrt{-\frac{g_{00}}{g^{11}}} g^{11} g^{00} \sqrt{g_{00}} \mathrm{~d} S_{1} \sim 0
$$

due to the facts that the electric field is finite on $r=r_{H}$ and $g^{11}\left(r_{H}\right)=0$. We conclude that the integral $I_{2}$ is null on the horizon.

The analysis of $I_{3}$ begins by taking $b=0$ in Eq. (22),

$E-a^{2} \partial_{1} K_{0}=C \frac{e^{\frac{v+\lambda}{2}}}{r^{2}}$,

and replacing it back into Eq. (40):

$$
I_{3}=-C \int_{r_{H}} A_{0} \sin \theta \mathrm{d} S_{1}
$$

Equation (42) is a second order differential equation for the field $E$, which may be homogeneous or non-homogeneous according to values of the constant $C$. In flat spacetime Eq. (42) becomes explicitly linear and we obtain 
two homogeneous solutions, $\frac{e^{ \pm \frac{r}{a}}}{r^{2}}\left(-1 \pm \frac{r}{a}\right)$, and one nonhomogeneous solution, $\frac{C}{r^{2}}$. In curved space-time the homogeneous solutions $E_{(h)}$ lead to $I_{3}=0$. Then from Eq. (34) we conclude that

$I_{1}=\int \sqrt{-g}\left[-g^{11} g^{00} E_{(h)}^{2}+g^{00}\left(a K_{0(h)}\right)^{2}\right] \mathrm{d}^{4} x=0$.

Since $g_{00}>0$ and $g_{11}<0$ in the region exterior to the horizon, each term in the square-brackets of $I_{1}$ is positivedefinite. Hence, the only possible solution to Eq. (44) is

$E_{(h)}=K_{0(h)}=0$ for $r \geq r_{H}$.

Therefore, the existence of the horizon imposes the requirement that the asymptotic solution $\left(r \gg r_{H}\right)$ of $E_{(h)}$,

$E_{(h)} \simeq-C_{1} \frac{e^{-\frac{r}{a}}}{r^{2}}\left(1+\frac{r}{a}\right)$,

must be null, i.e. $C_{1}=0$. Notice that the demonstration fails for the non-homogeneous solutions $E_{(N h)}$ because $I_{3}$ is different from zero in this case.

The non-homogeneous solution of Eq. (42) is

$E_{(N h)}=C \frac{e^{\frac{v+\lambda}{2}}}{r^{2}}$,

from which we verify that $K_{0(N h)}=0$ by using Eq. (23). If we replace this result in the Einstein equations (26) and (27) we obtain the Reissner-Nordström solution; in this case, the constant $C$ is the electric charge.

Therefore, we conclude that the exterior solution of the Einstein-Podolsky $\mathrm{BH}$ for $b=0$ is independent of the parameter $a$. This corroborates the no-hair theorem.

\subsection{Maxwell-Proca decomposition}

For $b=0$ the Podolsky field $A_{\mu}$ can be decomposed as

$A_{\mu}=A_{\mu}^{(M)}-A_{\mu}^{(P)}$

and these components satisfy the Maxwell and Proca equations:

$\nabla_{v} F^{\mu \nu(M)}=0$

$\nabla_{\nu} F^{\mu \nu(P)}=\frac{1}{a^{2}} A^{\mu(P)}$

where $F_{\mu \nu}^{(M)}=\nabla_{\mu} A_{\nu}^{(M)}-\nabla_{\nu} A_{\mu}^{(M)}$ and similarly for $F_{\mu \nu}^{(P)}$. Indeed, by rewriting Eq. (11) as

$\nabla_{v}\left[F^{\mu \nu}-a^{2}\left(\nabla^{\mu} \nabla_{\gamma} F^{\nu \gamma}-\nabla^{\nu} \nabla_{\gamma} F^{\mu \gamma}\right)\right]=0$ and by using Eqs. (48) and (49), it is trivial to verify that $F^{\mu \nu(M)}$ and $F^{\mu \nu(P)}$ satisfy Eq. (50). Besides, one can show that the Podolsky energy-momentum tensor,

$T_{\mu \nu}=T_{\mu \nu}^{M}+T_{\mu \nu}^{a}$,

can also be decomposed as $[30,31]$

$T_{\mu \nu}=T_{\mu \nu}^{(M)}-T_{\mu \nu}^{(P)}$,

where $T_{\mu \nu}^{(M)}$ and $T_{\mu \nu}^{(P)}$ are the Maxwell and Proca energymomentum tensors, given, respectively, by

$$
\begin{aligned}
T_{\mu \nu}^{(M)}= & \frac{1}{4 \pi}\left[F_{\mu \sigma}^{(M)} F_{\nu}^{\sigma(M)}+\frac{1}{4} g_{\mu \nu} F^{\alpha \beta(M)} F_{\alpha \beta}^{(M)}\right], \\
T_{\mu \nu}^{(P)}= & \frac{1}{4 \pi}\left[F_{\mu \sigma}^{(P)} F_{\nu}^{\sigma(P)}+\frac{1}{4} g_{\mu \nu} F^{\alpha \beta(P)} F_{\alpha \beta}^{(P)}\right. \\
& \left.+\frac{1}{a^{2}}\left(A_{\mu}^{(P)} A_{\nu}^{(P)}-\frac{1}{2} g_{\mu \nu} A^{\beta(P)} A_{\beta}^{(P)}\right)\right] .
\end{aligned}
$$

In this way, the Einstein-Hilbert Lagrangian together with

$\mathcal{L}_{m}=\mathcal{L}_{m}^{(M)}-\mathcal{L}_{m}^{(P)}$,

where

$\mathcal{L}_{m}^{(M)}=-\frac{1}{4} F^{\alpha \beta(M)} F_{\alpha \beta}^{(M)}$,
$\mathcal{L}_{m}^{(P)}=-\frac{1}{4} F^{\alpha \beta(P)} F_{\alpha \beta}^{(P)}+\frac{1}{2 a^{2}} A^{\mu(P)} A_{\mu}^{(P)}$,

lead to field equations which are equivalent to Eqs. (11) and (15) with $b=0$.

Hence, up to the negative sign in Eq. (54), the EinsteinPodolsky and Einstein-Maxwell-Proca systems are equivalent. This equivalence becomes clearer in the context of a static spherically symmetric $\mathrm{BH}$ where it has been shown independently by Bekenstein [11] and Teitelboin [12] that the Proca fields are null in the region exterior to the horizon. From Eq. (50) it follows that the solutions $F_{\mu \nu}^{(P)}$ correspond exactly to the homogeneous solutions $E_{(h)}$ to Eq. (42), both of which are consistent with $F_{\mu \nu}^{(P)}=E_{(h)}=0$ in the region $r \geq r_{H}$.

\section{Analysis of Podolsky black hole exterior solution with $b \neq \mathbf{0}$}

In this section we will study the exterior solution of a spherical $\mathrm{BH}$ in the presence of Podolsky electrodynamics assuming the parameter $b \neq 0$. Analogously to Sect. 3 , we contract Eq. (11) with $A_{\mu}$ and integrate over the region exterior to the $\mathrm{BH}$ horizon. This leads to

$I_{1 b}+I_{2 b}+I_{3 b}=0$ 
where

$$
\begin{aligned}
I_{1 b}= & \int \sqrt{-g} g^{00}\left[-g^{11} E^{2}+\left(a^{2}+2 b^{2}\right) K_{0}^{2}\right. \\
& \left.+2 b^{2}\left(g^{11} E\right)^{2}\left(\frac{v^{\prime}-\lambda^{\prime}}{r}\right)\right] \mathrm{d}^{4} x, \\
I_{2 b}= & \left(a^{2}+2 b^{2}\right) \int_{r_{H}} \sqrt{-g} g^{00} g^{11} K_{0} E \mathrm{~d} S_{1}, \\
I_{3 b}= & \int_{r_{H}} \frac{\sqrt{-g} A_{0}}{g_{00} g_{11}}\left[E-\left(a^{2}+2 b^{2}\right) \partial_{1} K_{0}\right. \\
& \left.+2 b^{2} S_{10}\right] \mathrm{d} S_{1} .
\end{aligned}
$$

Subtracting Eq. (27) from Eq. (26) implies

$$
\begin{aligned}
\frac{\lambda^{\prime}+v^{\prime}}{2 r}= & g^{00} g^{11}\left[a^{2}\left(\frac{K_{0}}{g^{11}}\right)^{2}+2 b^{2}\right. \\
& \left.\times\left(\left(\frac{K_{0}}{g^{11}}+\frac{2 E}{r}\right)^{2}+\frac{2 E^{2}}{r^{2}}\right)\right] .
\end{aligned}
$$

This result is then used to rewrite Eq. (58) as

$$
\begin{aligned}
I_{1 b}= & \int \sqrt{-g} g^{00}\left(-g^{11}\right)^{3}\left\{\left(\frac{E}{g^{11}}\right)^{2}+\frac{\left(a^{2}+2 b^{2}\right)}{\left(-g^{11}\right)^{3}} K_{0}^{2}\right. \\
& -\frac{4 b^{2}}{r} \frac{g_{00}^{\prime}}{g^{11} g_{00}} E^{2}+4 a^{2} b^{2} \frac{g^{00}}{\left(g^{11}\right)^{2}} r E^{2} K_{0}^{2} \\
& \left.+8 b^{4} g^{00} E^{2} r\left[\left(\frac{K_{0}}{g^{11}}+\frac{2 E}{r}\right)^{2}+\frac{2 E^{2}}{r^{2}}\right]\right\} \mathrm{d}^{4} x .
\end{aligned}
$$

If we assume $g_{00}^{\prime} \geq 0$ in the region exterior to the horizon then each term of $I_{1 b}$ is positive-definite. From a physical point of view, this hypothesis is the only acceptable one once $g_{00}^{\prime}<0$ is associated with repulsive gravity. Indeed, if there exists a sub-region $r_{1}<r<r_{2}$ exterior to $r_{H}$ where $g_{00}^{\prime}<$ 0 , then particles moving radially with low velocities would experience a repulsive force given by

$\frac{\mathrm{d}^{2} r}{\mathrm{~d} t^{2}} \simeq-c^{2} \Gamma_{00}^{1} \Rightarrow \frac{\mathrm{d}^{2} r}{\mathrm{~d} t^{2}} \simeq c^{2} g^{11} g_{00}^{\prime}$.

Thus, we would have a region where the particle is impelled to move away from the origin. An additional non-physical effect appearing if $g_{00}^{\prime}<0$ is the blue-shift of an electromagnetic wave emitted at $r_{1}$ and detected at $r_{2}$.

The next step is to show under which conditions the integrals $I_{2 b}$ and $I_{3 b}$ are null. In order to keep the trace of the energy-momentum tensor Eq. (32) evaluated at $r_{H}$ finite, $K_{0}$ must tend to zero at least as $\sqrt{g_{00}}$. Then from Eqs. (59) and (41) we have $I_{2 b} \sim I_{2} \sim 0$. On the other hand, the field equation (22) may be used to cast the integral $I_{3 b}$ in the form
$I_{3 b}=-C \int_{r_{H}} A_{0} \sin \theta \mathrm{d} S_{1}$.

By arguments identical to those in Sect. 3, we see that the homogeneous solutions $E_{(h)}$ of Eq. (22) $(C=0)$ imply $I_{3 b}=$ 0 . In this case, under the hypothesis $g_{00}^{\prime} \geq 0$ at $r \geq r_{H}$ we conclude from Eq. (62) that

$E_{(h)}=0$ for $r \geq r_{H}$.

Hence, the only solution of Eq. (22) that could possibly be non-null is the non-homogeneous solution $E_{(N h)}$, whose asymptotic behavior $\left(r \gg r_{H}\right)$ is of the type $C / r^{2}$. It is worth noticing that nothing guarantees the existence of an $E_{(\mathrm{Nh})}$ that is consistent with the boundary conditions imposed by a horizon. In the next section we will give an argument contrary to the existence of a non-null $E_{(N h)}$.

\section{Energy conditions}

In this section we will analyze the Podolsky BH in the light of the null energy condition and the weak energy condition (NEC and WEC, respectively). In particular, it is shown that the only non-trivial solution exterior to the $\mathrm{BH}$ horizon which does not violate NEC and WEC is the non-homogeneous solution $E_{(N h)}$ obtained with $b=0$.

We state that the energy-momentum tensor $T_{\mu \nu}$ respects the null (weak) energy condition if the inequality

$T_{\mu \nu} k^{\mu} k^{\nu} \geq 0$

holds for every null (timelike) vector $k^{\mu}[39,40]$. For the particular case of a diagonal $T_{\nu}^{\mu}$, the energy conditions are simply

$\rho+p_{i} \geq 0$ with $i=1,2,3$,

where $\rho \equiv T_{0}^{0}$ is the energy density and $p_{1} \equiv-T_{1}^{1}, p_{2} \equiv$ $-T_{2}^{2}$ and $p_{3} \equiv-T_{3}^{3}$ are the principal pressures. The WEC is satisfied if, besides Eq. (65), we have

$\rho \geq 0$.

Equations (29), (30) and (22) make it possible to rewrite Eq. (66) and part of Eq. (65) as

$$
\begin{aligned}
\rho= & -\frac{g^{00} g^{11}}{8 \pi}\left\{E\left(\frac{2 C e^{\frac{(v+\lambda)}{2}}}{r^{2}}-E\right)+a^{2} \frac{K_{0}^{2}}{g^{11}}\right. \\
& \left.+2 b^{2} g^{11}\left[\left(\frac{K_{0}}{g^{11}}+\frac{2 E}{r}\right)^{2}+\frac{2 E^{2}}{r^{2}}\right]\right\} \geq 0, \\
\rho+p_{1}= & -\frac{g^{00} g^{11}}{4 \pi}\left\{a^{2} \frac{K_{0}^{2}}{g^{11}}+2 b^{2} g^{11}\right.
\end{aligned}
$$




$$
\left.\times\left(\left(\frac{K_{0}}{g^{11}}+\frac{2 E}{r}\right)^{2}+\frac{2 E^{2}}{r^{2}}\right)\right\} \geq 0 .
$$

Equation (68) is satisfied in a region exterior to the horizon under two situations only, namely:

1. $K_{0}=0=b$, which leads to a Maxwell-like solution;

2. $K_{0}=0=E$, which implies a null field at $r \geq r_{H}$.

In the first case, we also have $\rho+p_{2} \geq 0$ and the energy density is given by

$\rho=-\frac{g^{00} g^{11}}{8 \pi} E^{2}$

which is positive-definite. Hence, for $b=0$ we conclude that the only solution compatible with both NEC and WEC is $E=E_{(N h)}$ given by Eq. (46).

In the second situation (where $b \neq 0$ ), the only solution satisfying NEC and WEC is the trivial solution $E=0$. This result disfavors the existence of a non-null solution $E_{(\mathrm{Nh})}$ in the region exterior to the horizon.

Finally, it is interesting to note that conditions (67) and (68) can be used to constrain some physical configurations even out of the context of BH. For instance, from Eq. (67) we see that purely homogeneous solutions (i.e., those with $C=0$ ) will always have a negative energy density. For this reason, they are physically disfavored.

\section{Final remarks}

In this work, we have studied black holes in the presence of a matter field given by Podolsky electrodynamics. The paper is composed of three main parts: in the first one, we presented the generalization of Podolsky electrodynamics to curved space-time; in the second part, we analyzed static spherically symmetric solutions exterior to Podolsky $\mathrm{BH}$ horizon; in the third part, these solutions were scrutinized in the light of the null and weak energy conditions.

The generalization of Podolsky electrodynamics to curved space-times gives rise to two possible types of Lagrangian. The first one is obtained by performing the minimal coupling prescription in Eq. (1) which implies $b=0$ in Eq. (8). The second possible Lagrangian is built from Utiyama's approach [34] (meaning $b \neq 0$ in Eq. (8)). This was shown to be equivalent to the first Lagrangian up to non-minimally coupled terms depending on the contraction of the Riemann tensor and the field strength. This study has its importance not only at the classical level but also in the quantum context. For instance, we can speculate if the replacement of Maxwell theory by Podolsky's would (or would not) help to control ultraviolet 1-loop divergences that are present in the Einstein-Maxwell case [38]. It is worth emphasizing that something similar happens in flat space-time where Podolsky electrodynamics guarantees the finiteness of the electron self-energy and vertex correction at 1-loop [41].

The exterior solutions were analyzed for two distinct cases, namely those obtained by taking $b=0$ and $b \neq 0$ in the equations of motion. The only non-trivial solution for the electromagnetic field when $b=0$ was shown to be Maxwell's solution, which leads to the Reissner-Nordström $\mathrm{BH}$. We also verified that the Einstein-Podolsky system can be decomposed into an Einstein-Maxwell-Proca-like system, recalling that the Proca field is null on the $\mathrm{BH}$ exterior region $[11,13]$. Thus, we concluded that the no-hair theorem is satisfied when $b=0$ in two different ways. For the case where $b \neq 0$, we have verified that the homogeneous (asymptotically massive) solutions $E_{(h)}$ are null in the region exterior to the $\mathrm{BH}$ horizon under the physical hypothesis $g_{00}^{\prime} \geq 0$.

Podolsky electrodynamics preserves $U(1)$ gauge invariance. Therefore, the absence of propagation of one of the Podolsky modes in the region exterior to the horizon is directly associated to the fact that this is a massive mode; the lack of a Podolsky propagating mode is not related to the theory's gauge invariance.

In the last part, we verified that the only exterior solution consistent with the weak and null energy conditions is Maxwell's solution, i.e. $E_{(N h)}$ with $b=0$. Therefore, any possible non-Maxwellian solution (a solution with hair - e.g. $E_{(N h)}$ with $b \neq 0$ ) necessarily violates NEC and WEC. Moreover, it was shown that any purely homogeneous exterior solution to the Podolsky $\mathrm{BH}$ has a negative-definite energy density. Particularly, this can be verified for the case $b=0$ in the context of the Maxwell-Proca decomposition; then $T_{\mu \nu}^{(M)}$ is null, since $E_{(N h)}=0$, leading to $T_{\mu \nu}=-T_{\mu \nu}^{(P)}$. This decomposition shows that our Proca field is a ghost field.

The conclusion is: under reasonable physical hypotheses, the static spherically symmetric Podolsky BH satisfies the no-hair theorem. However, for $b \neq 0$, solutions with hair are not mathematically excluded. In a future work, it would be interesting to investigate if these solutions exist and what properties they possess.

Acknowledgements RRC is grateful to R. H. Brandenberger for the kind hospitality extended to him at McGill and CAPES-Brazil (Grant 88881.119228/2016-01) for financial support. BMP acknowledges CNPq-Brazil for partial financial support. LGM is grateful to CNPq-Brazil (Grant 112861/2015-6) for financial support. The authors thank E. M. de Morais for useful discussions during the early stages of this work.

Open Access This article is distributed under the terms of the Creative Commons Attribution 4.0 International License (http://creativecomm ons.org/licenses/by/4.0/), which permits unrestricted use, distribution, and reproduction in any medium, provided you give appropriate credit to the original author(s) and the source, provide a link to the Creative Commons license, and indicate if changes were made.

Funded by SCOAP $^{3}$. 


\section{References}

1. A. Einstein, Sitzungsber. Preuss. Akad. Wiss. Berlin 844 (1915)

2. K. Schwarzschild, Sitzungsber. Preuss. Akad. Wiss. Berlin 7, 189 (1916)

3. H. Reissner, Annalen der Physik 50, 106 (1916)

4. G. Nordstrm, Verhandl. Koninkl. Ned. Akad. Wetenschap. Afdel. Natuurk. Amsterdam 26, 1201 (1918)

5. R.P. Kerr, Phys. Rev. Lett. 11, 237 (1963)

6. E. Newman, A. Janis, J. Math. Phys. 6, 915 (1965)

7. E. Newman, E. Couch, K. Chinnapared, A. Exton, A. Prakash, R. Torrence, J. Math. Phys. 6, 918 (1965)

8. W. Israel, Phys. Rev. 164, 1776 (1967)

9. W. Israel, Commun. Math. Phys. 8, 245 (1968)

10. B. Carter, Phys. Rev. Lett. 26, 331 (1971)

11. J.D. Bekenstein, Phys. Rev. D 5, 1239 (1972)

12. C. Teitelboim, Phys. Rev. D 5, 2941 (1972)

13. J.D. Bekenstein, Phys. Rev. D 5, 2403 (1972)

14. S.W. Hawking, Commun. Math. Phys. 25, 167 (1972)

15. A.E. Mayo, J.D. Bekenstein, Phys. Rev. D 54, 5059 (1996)

16. T.P. Sotiriou, V. Faraoni, Phys. Rev. Lett. 108, 081103 (2012)

17. K.G. Zloshchastiev, Phys. Rev. Lett. 94, 121101 (2005)

18. C.A.R. Herdeiro, E. Radu, Int. J. Mod. Phys. D 24, 1542014 (2015)

19. C.A.R. Herdeiro, E. Radu, H. Rnarsson, Class. Quantum Gravity 33, 154001 (2016)

20. W.E. East, F. Pretorius, Phys. Rev. Lett. 119, 041101 (2017)

21. D. Gottlieb, R. Hojman, Nuovo Cim. B 80, 62 (1984)

22. C. Vuille, J. Ipser, J. Gallagher, Gen. Relativ. Gravit. 34, 689 (2002)

23. N. Rosen, Found. Phys. 24, 1689 (1994)
24. Y.N. Obukhov, E.J. Vlachynsky, Ann. Phys. 8, 497 (1999)

25. M. Toussaint, Gen. Relativ. Gravit. 32, 1689 (2000)

26. F. Bopp, Ann. Phys. 430, 345 (1940)

27. B. Podolsky, Phys. Rev. 62, 68 (1942)

28. D.S. Kaparulin, S.L. Lyakhovich, A.A. Sharapov, Eur. Phys. J. C 74, 1 (2014)

29. R.R. Cuzinatto, C.A.M. de Melo, P.J. Pompeia, Ann. Phys. 322, 1211 (2007)

30. A.E. Zayats, Ann. Phys. 342, 11 (2014)

31. A.E. Zayats, Phys. Rev. D 94, 105026 (2016)

32. Z. Haghani, T. Harko, H.R. Sepangi, S. Shahidi, Eur. Phys. J. C 77, 137 (2017)

33. R.R. Cuzinatto, E.M. de Morais, L.G. Medeiros, C.N. de Souza, B.M. Pimentel, Eur. Phys. Lett. 118, 19001 (2017)

34. R. Utiyama, Phys. Rev. 101, 1597 (1956)

35. R.R. Cuzinatto, C.A.M. de Melo, L.G. Medeiros, P.J. Pompeia, Int. J. Mod. Phys. A 26, 3641 (2011)

36. C.A. Bonin, R. Bufalo, B.M. Pimentel, G.E.R. Zambrano, Phys. Rev. D 81, 025003 (2010)

37. I.T. Drummond, S.J. Hathrell, Phys. Rev. D 22, 343 (1980)

38. S. Deser, P. van Nieuwenhuizen, Phys. Rev. D 10, 401 (1974)

39. S.W. Hawking, G.F.R. Ellis, The large scale structure of spacetime (Cambridge University Press, Cambridge, 1973)

40. R.M. Wald, General Relativity (University of Chicago Press, Chicago, 1984)

41. R. Bufalo, B.M. Pimentel, G.E.R. Zambrano, Phys. Rev. D 83, 045007 (2011) 